\title{
Noise Reduction using Kalman Filter
}

\author{
Er. Gaurav Badhwar \\ Student, Electronics and Communication Department,, \\ Punjabi University, Patiala, Punjab, India \\ Er. Sweety Badhwar \\ Electronics and Communication Department, \\ Punjabi University, Patiala, Punjab, India \\ Dr. Amandeep Singh Sappal \\ Asst. professor, Electronics and Communication Department, \\ Punjabi University, Patiala, Punjab, India
}

\begin{abstract}
In speech communication systems, it is found that speech signals easily get affected by noise and tamper the system accuracy and performance. Before processing a speech signal, it is very important to filter out the noise from corrupted speech signal to enhance the accuracy and the performance of communication systems. It is also important to enhance the quality of the speech and also to enhance the listening ability. But in practice, it is very complicated to filter out the noise from the desired speech signal to obtain noise free speech signal all time for the speech communication system. In the last few years, several speeches filtering algorithms have been introduced in order to filter out the noise from the desired speech signal.

In this paper, the problem of speech enhancement is discussed when a corrupted speech signal with an additive white noise is the only information available for processing. The main idea of the paper is to use the Kalman filtering technique to estimate the future clean samples from the first one in an iterative way. The simulation uses mean square error as a tool to realize the similarity and minimum errors between estimated signal and true signal.
\end{abstract}

Keywords - Kalman filter, Speech enhancement, Speech communication, Additive white noise.

\section{INTRODUCTION}

SPEECH is the vocalized form of communication between human civilizations It is the most common form of communication between humans.

Enhancement implies improvement and increase in quality of something. Similarly, speech enhancement means improvement of quality and clarity of corrupted speech signal by using several processing techniques. So its aim to filter out noise from the input signal and to improve the performance and the accuracy of voice communication systems when input signals are corrupted by additive noise[3]. But in practice it is an extremely complicated issue for two reasons. First, the nature and qualities of the noise indicators that change significantly with time. So it is additionally complicated to discover algorithms that truly work in these real time situations. Second, the execution measure can likewise be characterized contrastingly for every requisition.

There are many criteria for measuring performance of speech enhancement. The quality of the enhanced signal and the level of residual noise in that signal are two main criteria for measuring speech enhancement performance. The quality is measure of clarity of enhanced signal. It is very complicated to fulfill these criteria in the meantime.

There are different speech enhancement methods proposed for noise lessening and to enhance the speech quality and clarity. Iterative Wiener filtering analysis, Kalman filtering analysis, Linear Predictive Coding (LPC) analysis and Signal Subspace method are some methods for Speech Enhancement. The execution of these methods depends upon the quality and comprehensibility of the input speech signal. The least mean square error is generally focused of most methods. Enhancement systems may be classified as single channel and double channel or multi-channel enhancement systems. When there is one and only secured channel is accessible single channel enhancement systems are preferred. This paper focus is on the single channel enhancement system. 


\section{PROBLEM STATEMENT AND AIM}

\section{A. Problem Statement}

The speech signal is contaminated by white noise with discrete time observation signal is represented as: $y(n)=x(n)+v(n)$

(1)

where $x(n)$ is clean speech sequence and $v(n)$ is additive white noise.

\section{B. Objectives}

The primary objective of this paper was to the reconstruction and enhancement of the speech signal, corrupted by additive white noise, with Kalman filtering technique, using the MATLAB simulator.

\section{KALMAN FILTER ALGORITHM}

In 1960, Rudolf E. Kalman published his paper on Kalman filter which provide a recursive solution to the discretedata linear filtering problem[1]-[2]. Over the last forty years, Kalman filter has been the subject of extensive research and application, such as target tracking, noise cancellation and many other dynamic systems.The Kalman filter algorithm is based on minimising the mean square error recursively[4]. There are many tools to compare the actual signal with estimated signal such as cross-correlation and least square [7]. The major advantage of Kalman filter is that for computing the new state estimate in each cycle, only the previous state estimate and the new input data are required, which results in a low memory requirement [5]. It is found that Kalman filtering method has significantly better performance than the Wiener filtering method in noise cancellation[6].

Kalman filter is an optimal estimator which infers parameters of interest from indirect, inaccurate and noisy observations. Two linear difference stochastic equations used to describe steady state Kalman approach to digital filtering signal $\left\{w_{n}\right\}$ are:

$$
\begin{aligned}
& x_{n}=F^{x_{n-4}}+G w_{n-1} \\
& y_{n}=H^{x_{n}}+v_{n}
\end{aligned}
$$

Process of Signal Generation is described by the first equation and signal measurement is described by the second equation. The process and measurement noises are represented by the random variables $\left\{w_{n}\right\}$ and $\left\{v_{n}\right\}$ respectively.It is assumed they are mutually exclusive and white noise variables with normal probability distribution.

The process noise covariance matrix is given by

$$
E\left[w_{n}^{*} W_{1}\right]=\varphi_{R A} p\left(w_{n}\right) \sim W(Q, \varphi)
$$

The Measurement noise covariance matrix is given by

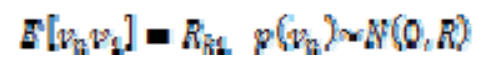

For Simplification, covariance matrix of the process noise and the measurement noise are assumed to be constant but in practice, they might change with time step of time series.

When the process noise is not present, the matrix F shows the relationship between the state at a time step $n-1$ to 
the state at time step $n$. The matrix $\boldsymbol{F}$ might change with time, but it is assumed to be invariable. The state $x_{n}$ is related to the measured value of $y_{n}$ by the matrix $H$ which is also assumed to be constant. The Kalman filter estimates the process state at step ${ }^{n}$ and then obtains feedback in the form noisy measurements. Therefore Kalman filter equations are divided into two groups. The first is the time update(prediction) or process update equations and the second one is the measurement update(correction) equations.

The process update equations are

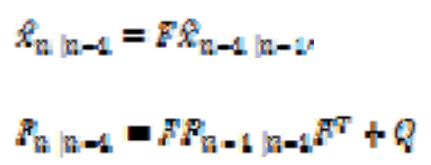

The measurement update equations are

$$
\begin{aligned}
& E_{\mathrm{n}}=F_{\mathrm{n} \mid \mathrm{n}-1} R_{\mathrm{n}}^{\gamma}\left[R_{\mathrm{n}} P_{\mathrm{n}-1} R_{\mathrm{n}}^{\gamma}+R\right]^{-1}, \\
& x_{n, n}=x_{n, n-1}^{q_{n}}+K_{n}\left(y_{n}-R_{n} x_{n, n-1}\right) \\
& P_{n \mid n}=\left[I-K_{n} F_{n}\right] P_{n=1}
\end{aligned}
$$

Time update equations projects the current state and error covariance estimates to obtain the prior estimates for the succeeding time step. Matrix $F_{\mathrm{n}} \mathrm{n}-\mathbf{4}$ represent a priori estimate of error covariance

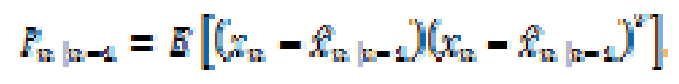

and Matrix $\boldsymbol{P}_{\mathrm{n}}$ in represents a posteriori estimate of error covariance

$$
F_{n, n}=E\left[\left(x_{n}-x_{n n}^{*}\right)\left(x_{n}-x_{n n}^{*}\right)^{7}\right]
$$

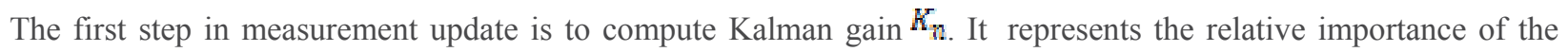
error $y_{n}$ with respect to the prior estimate $\hat{n}_{n} n_{n}-1$ the next step is to generate the posteriori state estimate $i_{n} n_{n}$ by linear combination of priori estimate $\hat{n}_{\mathrm{n}} \mathrm{m}-\mathbf{t}_{\text {tand }}$ a weighted difference between the actual value of measurement $y_{n}$ and measurement prediction.

$$
f_{n=1}=H_{n} x_{n} n_{n=4}
$$

The last step in measurement updates is to compute a posteriori error estimate via equation(10). The process repeated with the previous a posteriori estimates used to predict the new a priori estimates as recursive solution of one of the attractive nature of Kalman filter.

\section{SIMULATION RESULTS}

To achieve the aim of this paper the simulation source code is written in MATLAB to employ Kalman filter in speech recorded. In this paper, a speech signal added with white Gaussian noise is selected for simulation. The tool used to realize the similarity and minimum errors between estimated signal and true signal is mean square error. 

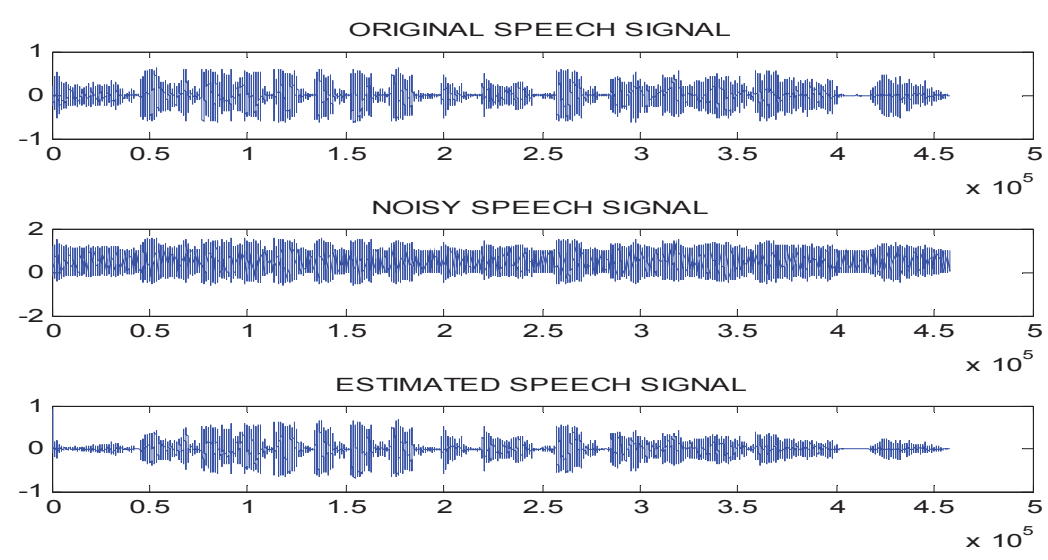

Fig 1. shows Original signal, Noisy signal and Estimated signal.

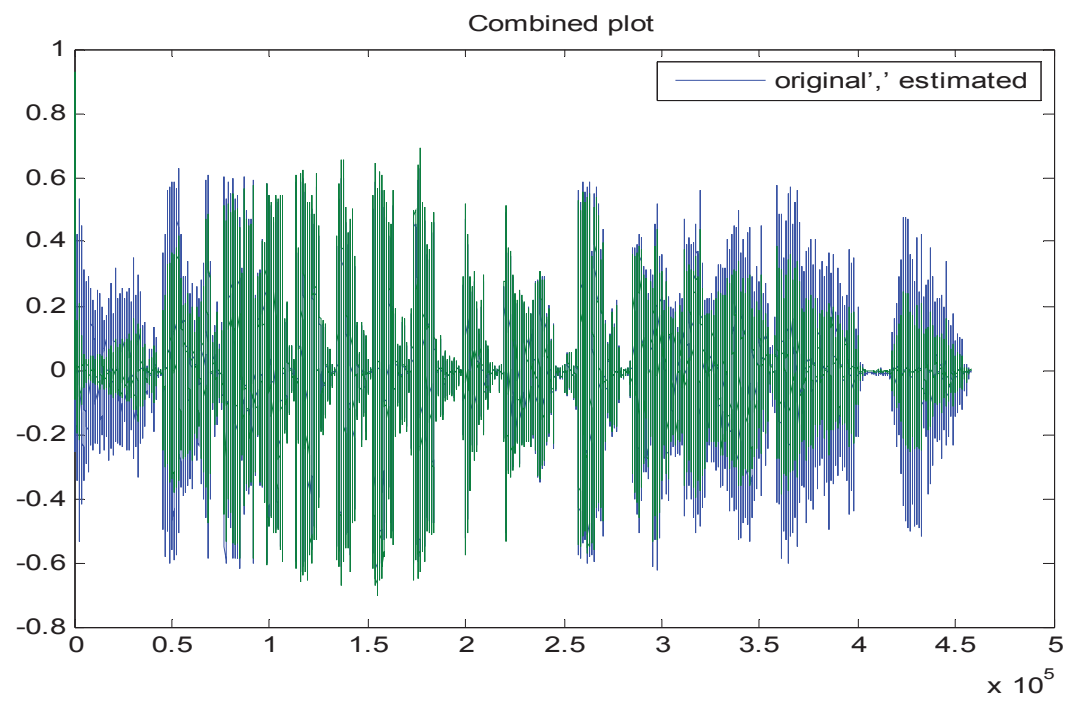

Fig 2. shows Combined plot of Original and Estimated signal.

Above figure shows that with passage of time, kalman filter produces a more and more precise estimation of speech signal from input signal by filtering out white noise. 


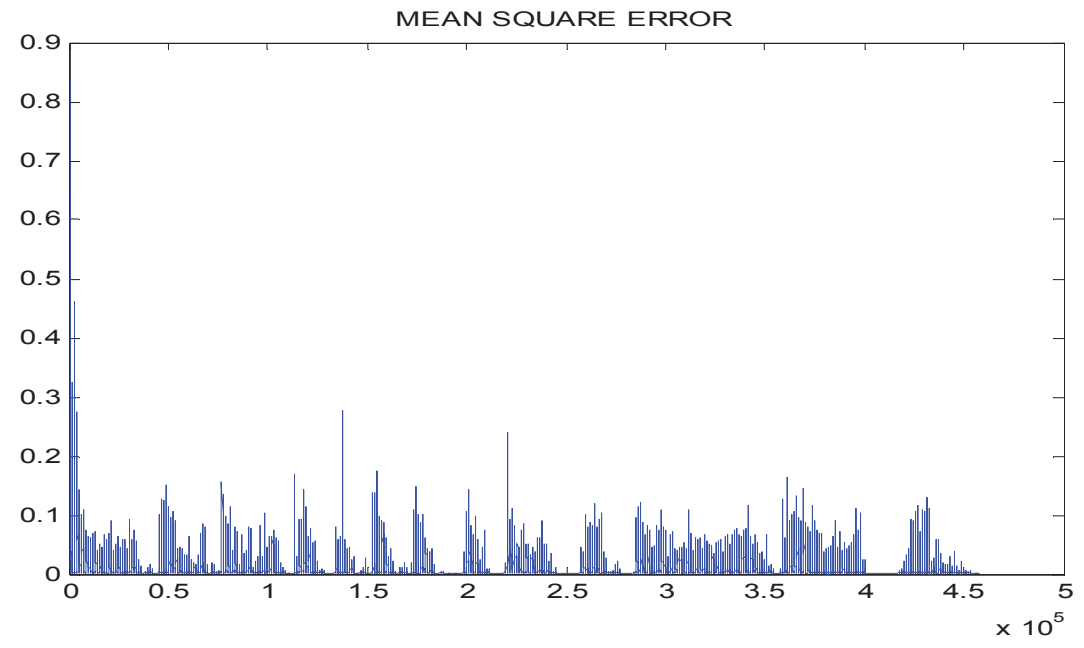

Fig 3. Mean Square Error.

Above figure shows that there is a continuous reduction in mean square error with time.

\section{CONCLUSION}

Speech enhancement using Kalman filter technique is performed when a corrupted speech signal with an additive white noise is the only information available for processing. The simulation results show that the Kalman filtering technique produced an excellent estimate of future clean samples from the first one in an iterative way. Moreover, testing is done by mean square error during simulation to check the level of similarity between input and output speech signal. With the increasing iterations i.e. with the passage of time, Kalman filter presents a more and more precise estimation of speech signal from the input signal by filtering out white noise. Also with the increasing iterations, it has been observed that the mean square error decreases continuously. On the whole this paper has done well to achieve and solve the problem statement.

\section{REFERENCES}

[1] R.E.Kalman, "A new approach to linear filtering and prediction problems", Journal of Basic Engineering 82D, 1960, pp. 34-45.

[2] Kalman, R. E., and Buch, R. S., " New Results in Linear Filtering and Prediction Theory" ASME journal of basic engineering, 1961, pp. 95-108.

[3] Prasad, G., and Surender, "A Review of Different Approaches of Spectral Subtraction Algorithms for Speech Enhancement", Current Research in Engineering, Science and Technology (CREST) Journals. Vol. 01. Issue 02, 2013, pp. 57-64.

[4] S. Haykin, Adaptive Filter Theory, 4th Edition, Prentice Hall,

[5] E.W. Kamen, and J.K. Su, Introduction to Optimal Estimation, Springer, UK, 1999,SA, 2002.

[6] K.K.Paliwal and A.Basu, " A Speech Enhance-ment Method Based on Kalman Filtering", n Proc. ICASSP'87, 1987, pp.177-180.

[7] Eng. Alaa K. Satti Salih, "Speech enhancement using kalman filter in communiction", International Journal of Mathematical, Computational, Physical, Electrical and Computer Engineering., Vol. 8, No:3, 2014.

[8] S. Cannot, D. Burchtein and E. Weinstein, "Iterative and Sequential Kalamn Filter-Based Speech Enhancement algorithms", IEEE Trans. on Speech and audio Proce.rsing. pp.373-385, July 1998

[9] J. Vermaak, M. Niranjan and S. J. Godsill, "Compaing Solution Methodologies for Speech Enhancement within a Bayesian Framework, Technical Repon CUED/FINFENGRR329, Cambridge University Engineering Department, August 1998. 